\title{
College and University Library Statistics, I943
}

$\mathrm{T}$ HE 1942-43 PLAN of using volunteers seemed impractical for another year because of the manpower shortage in libraries. The budgetary situation made a full-time statistical assistant out of the question. Work in the Public Library
Division was therefore reorganized to make it possible for the assistant, Kathryn P. Mier, to divide her time between that division and statistics, for the two large compilations only-college and university libraries and public libraries.

\section{DEFINITIONS USED IN COMPILING SALARY FIGURES}

\section{Faculty Members}

I. "Faculty members" includes instructional and organized research staffs, extension service staff, and general administration officers (excluding clerical workers), and professional library staff, all reduced to fulltime equivalent.

\section{Administrative Office Assistant}

2. An administrative office assistant is a person such as the business manager or secretary to the chief librarian or director who performs work of a highly specialized nature but not that requiring training and skill in the theoretical or scientific parts of library work.

Formal education or experience equivalent to that required of such workers in a large business organization should be considered as prerequisite.

\section{Department Head}

3. A department head is a member of the professional staff directly responsible to the chief librarian or director, associate or assistant chief librarian, and in charge of a major division of the library organization which has its own staff and definite responsibilities. (Salaries of an associate or assistant chief librarian serving as department head should not be included here.)

The department head must meet the qualifications of a professional assistant as defined in definition 4 .

\section{Professional Assistant}

4. A professional assistant is a member of the professional staff performing work of a professional grade which requires training and skill in the theoretical or scientific parts of library work as distinct from its merely mechanical parts and includes all the professional staff except the chief librarian or director, associate or assistant chief librarian, department heads, division heads, and heads of school, college, departmental, and laboratory school libraries.

To be classed as a professional assistant, the person must hold a professional position which requires the following educational background:

a. At least a bachelor's degree which includes one year of professional library education in the four years which lead to the bachelor's degrees; or

b. An informal education considered by the librarian as the real equivalent of four years of college work, plus five years' experience in a library of recognized professional standing. (This provision is to take care of those already in the profession who are performing duties which require a knowledge of books and library technique as taught in a library school.) 


\section{Subprofessional Assistant}

5. A subprofessional assistant is a person who performs, under the immediate supervision of professional staff members, work largely concerned with the higher routine processes which are peculiar to library work and which require some knowledge of library procedure. (No assistant paid on an hourly basis should be included here.)

A subprofessional assistant should have had at least brief elementary training in library work as taught in a library school summer session or a training class.

\section{Clerical Assistant}

6. A clerical assistant is a person such as a typist, etc., who performs, under immediate supervision, processes which may require experience, speed, accuracy, and clerical ability of a high order but do not require knowledge of the theoretical or scientific aspects of library work.

High school graduation is presupposed for this classification.

\section{Others}

7. "Others" includes those devoting either full or part time to the most simple clerical or manual tasks which involve no responsibility or independent judgment such as the work performed by pages, shelvers, and beginning workers.

\section{Student Assistant}

8. A student assistant is a student paid on an hourly basis, directly from the library budget, working in the library in any capacity except in janitorial service. (All student assistants on an hourly basis should be included here and in no case should they be entered elsewhere. No assistants on N.Y.A., W.P.A., or other federal, state, or local projects should be included here or in other personnel groups.)

\section{School or College Library in a University}

9. A school or college library, in a university system, is a collection of books related to the work of the particular school or college and administered either separately by the school or college or as a part of the university library. (Include group libraries such as biological sciences and social sciences here.)

\section{Departmental Library}

Io. A departmental library is a collection attached to a department of instruction which forms a part of a college administration. Such a collection may be housed either within or without the central building.

\section{Vacations and Special Holidays}

I I. "Vacations" and "special holidays" are reckoned in number of working days allowed with pay. "Special holidays" include Christmas and Easter vacations, legal holidays, etc. 


\section{College and University Library General and Salary Statistics (Group I)*}

Library

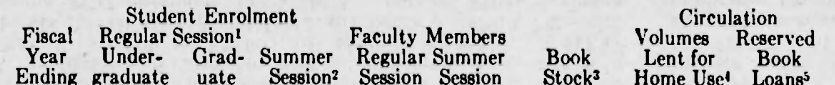

Library Operating Expenditures Last Fiscal Year $\begin{gathered}\text { Total College } \\ \text { Sinding } \\ \text { on University } \\ \text { Expenditureg } \\ \text { Last Fiscal }\end{gathered}$

University of Alabama

University of Arizona.......

Bavlor University

University of California (Berkeley)

University of Chicago

University of Cincinnati... Colorado State Agriculturai College. Dumbia University

University of Georgia.
Harvard University2i

Howard University

State University of Io......

lowa State College ..............

University of Kansas...................

University of Michigan.

University of Minnesota.

Mount Holyoke Colleget

University of Nebraska.

University of North Carolina

orthwestern University.

University of Oregon ....
Oregnn State Agricult ual College.

ennsylvania State College
University of Pennsylvanis

University of Pittsburgh.

Princeton Universitv.......

South Dakota State College .............

Southern Methodist

Syracuse University
University Libraries

University Libraries . . . . . . . . . .

Temple University.........

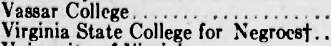

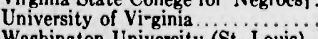

Washington University (St. Louis)

Wayne Univers

University of Wyoming................... $30 . \mathrm{30 \textrm {Je } e 4 3}$

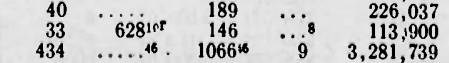

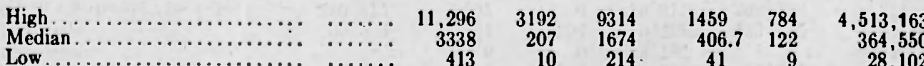

1 As of third week, fall term 1942. 2 Includes graduate students. 3 Includes volumes in all agencies. 4 Ex-
cludes overnight loans and all loans of reserved books. 5 Includes overnight loans. 6 Excludes capital outtay and auxiliary enterprises such as dormitory, athletics, etc. 7 Open shelves, includes overnight loans only. 8 Not

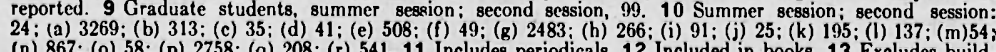
ing operation and maintenance: $\$ 2679$; (a) $\$ 2139$; (b) \$36; (c) $\$ 74,882$; (d) $\$ 11,448$; (e) $\$ 115$; (f) $\$ 297$; (g) $\$ 3865 ;$
(h) $82,567.14$ Not available. 15 Intersession, 1568 ; (a) $65 ;$ (b) 1729.16 On a four-quarter basis. 17 Excludes (h) $82,567.14$ Not available. 15 Intersession, 1568; (a) 65 ; (b) 1729.16 On a four-quarter basis. 17 Exclude
\$20.931 outstanding orders. 18 Does not include Teachers College. 19 Graduate and professional students clates periodicals, binding, and rebinding. 23 Includes graduate students enrolled for both summer sessions
cludes
121 ; second summer session, 1479. 24 Except as otherwise noted, data for Harvard College collection and 15

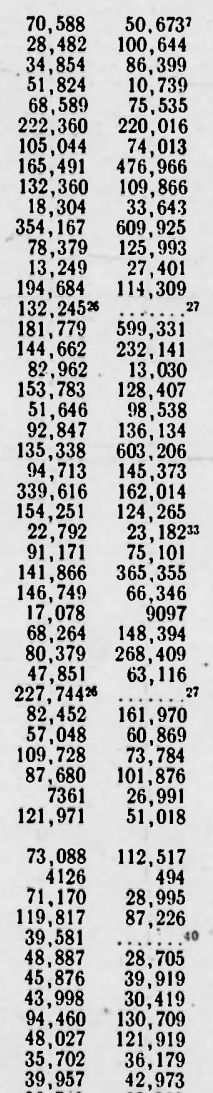

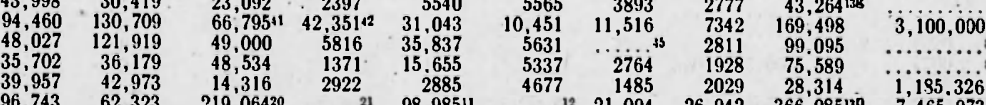

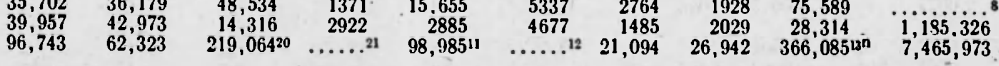
$\begin{array}{rrrrrrrrr}354,167 & 603,206 & 271,781 & 55,584 & 98,98547 & 35,081 & 42,420 & 483,379 & 11,361,722 \\ 80,379 & 92,882 & 48,775 \frac{1}{2} & 6585 & 30,4653 & 4871 & 4036 \frac{1}{96,831} & 2,686,298 \\ 4126 & 494 & 7100 & 1106 & 985 & 86 & 40 & 15,742 & 106,747\end{array}$ special collections. 25 For whole university library, \$175,190; (a) $\$ 40,610.26$ Includes reserved book loans, 27 In chools. 31 Total for firt and second summer sesions. 32 Includes General. High School libraries. 33 Partial count only. 34 Estimated. 35 Includes total of 189 . for both sessions for stu-

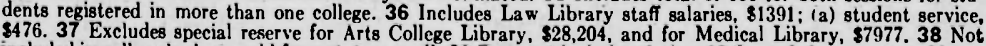
included in college budget; paid from state payroll. 39 Does not include salaries. 40 Open shelves, no record kept

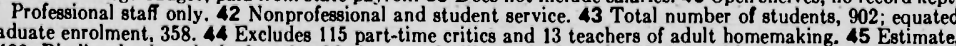
basis. 47 inding by board of education bindery not in library budget. 46 Undergraduate school on year-roun 1000. * Includes colleges and universities with enrolments of 1000 and over. 
College and University Library General and Salary* Statistics (Group I)

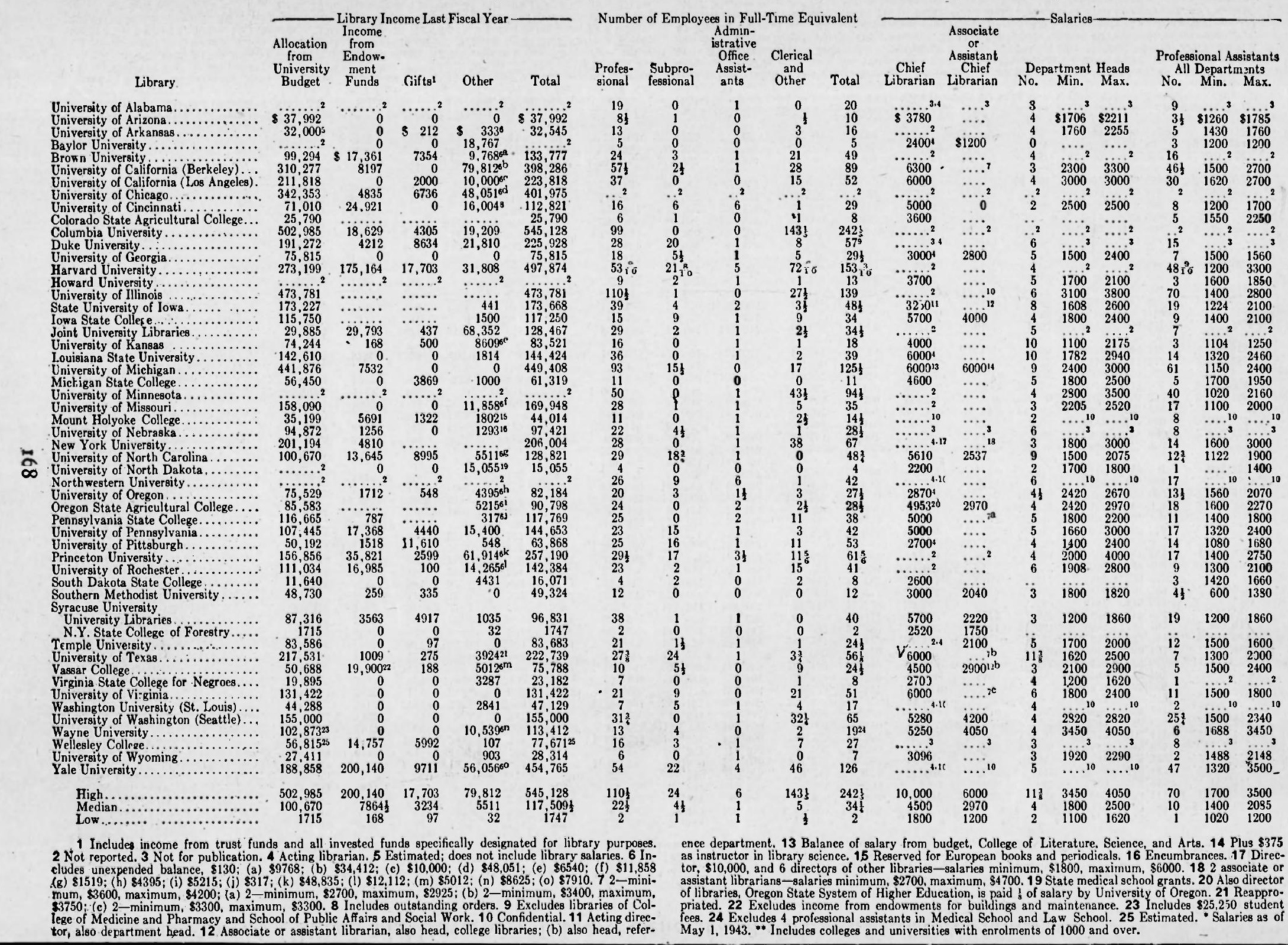


College and University Library General and Salary* Statistics (Group I)**

Number of Months $\begin{gathered}\text { Hours per Week } \\ \text { for Which. }\end{gathered}$ Rumber of Days Allowed
With Pay As:

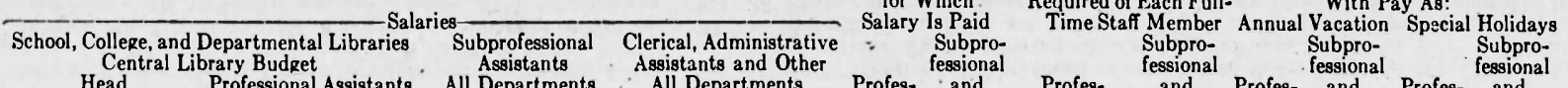

Library

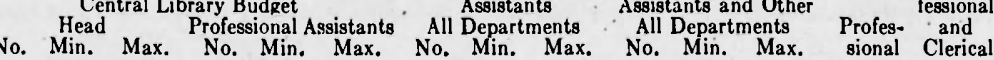

Profes- fessional Profes- ansional and Profes- and

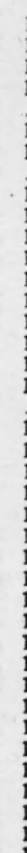

ersity of Arizona. Baylor University... Brown University

niversity of California (Berkeley niversity of California (Los Angeles). University of Cincinnat. Colorado State Agricultural College. Columbia University University of Georgia Harvard University. Howard University..... State University of lowa........... owa State Colleg University of Kanaas. University of Kansas.............
Louiciana State University
University of Michigan University of Michigan. nivereity of Minnesota.

Mount Holyoke College.

University of Nebraska.
New York University.....

New York University...................

University of North Dakota. University of Oregon

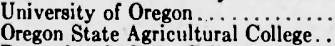
Pennsylvania State College. University of Pittsburgh ... Univer sity of Rochester South Dakota State College.
Southern Methodist University. Syacasuse University.

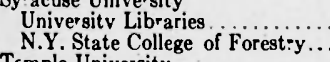

University of Texas.

irginia State College for Negrou. Wnivergity of Virginia .......................... Washington University (St. Louis)
University of Washington (Seattle) Wayne University

University of Wyoming $\begin{array}{lcc}3 & \ldots \ldots^{1} & \ldots{ }^{1} \\ 3 & \$ 1540 & \$ 1925\end{array}$

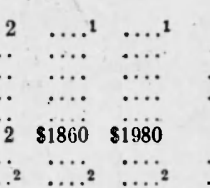
$\begin{array}{ll}2700 & 2900 \\ 1500 & 2300\end{array}$ 1. …

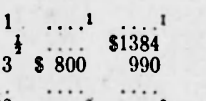
$960 \quad 2000$ $4 \frac{1}{2} \quad 340 \quad 1700$ $\begin{array}{llllll}201 & 1300 & 2600 & 12 & 1400 & \ldots 000 \\ 1 & \ldots & 2400 & 10 & 1260 & 1716\end{array}$ $\begin{array}{rrr}6 & 72^{2} & 1800^{2} \\ 3 & 950 & 1800 \\ 11 & 1200 & 1914\end{array}$ $\begin{array}{lll}11 & 1200 & 1914 \\ 14 & 1400 & 325\end{array}$ $\begin{array}{lll}10 & \ldots n^{2} & \cdots^{2}\end{array}$ $7 \quad 1128 \quad 1600$ $5 \quad 1200 \quad 1980$ $7 \quad 750 \quad 2730$

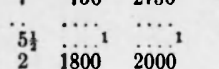
.... $\cdots$. … 1450

$$
\begin{aligned}
& \text { High.... } \\
& \text { Median. } \\
& \text { Low..... }
\end{aligned}
$$

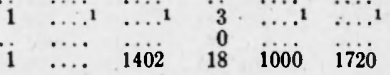$$
\begin{array}{lll}
22 & \cdots & 2 \\
30 & 1080 & 2580 \\
15 & 1200 & 1560
\end{array}
$$

1 Not for publication. 2 Not reported. 3 Exception-Morrison Library subprofessional has whole summer;

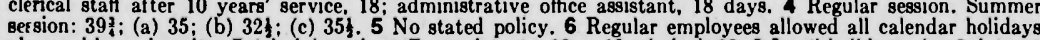
otserved by university. 7 Administrative office assistants. i2 or 18 ; cercical 12. 8 Lepal holidays plus 2 days at Chrietmas. 911 working days for each month worked. 10 Confidential, 11 Since April 1943.12 After 3 months'
Bervice, $6 ; 6$ months', 12; 2 years', 18; 3 years', 24 . 13 Plus $\$ 195$ for summer session, 14 All staff menibers with

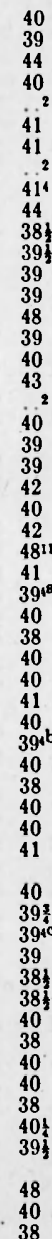

Sabbatical
Leave None None None None
None $\ldots \ldots \ldots .^{2}$ Librarian None None All None None None Director Librarian Librarian None None..... None...
Allis All1" Libr. \& Asst. Libr. None None.....? …..... None Nons Librarian None None
None Librarian'21 Librarian'21
Professional
None None

..............

rank of assistant professor or above. 15 Plus luncheons. 16 Clerical, 11. 17 Except one assistant teaching library science, 101.1812 for first 5 years, 15 for 5 to 10 years, 18 for more than 10 years. 19 Part-time assistant, $\$ 600$ research librarian. *Salaries as of May 1,1943 . *"Includes colleges and universities with enrolments of 1000 and 
College and University Library General and Salary Statistics (Group II)*

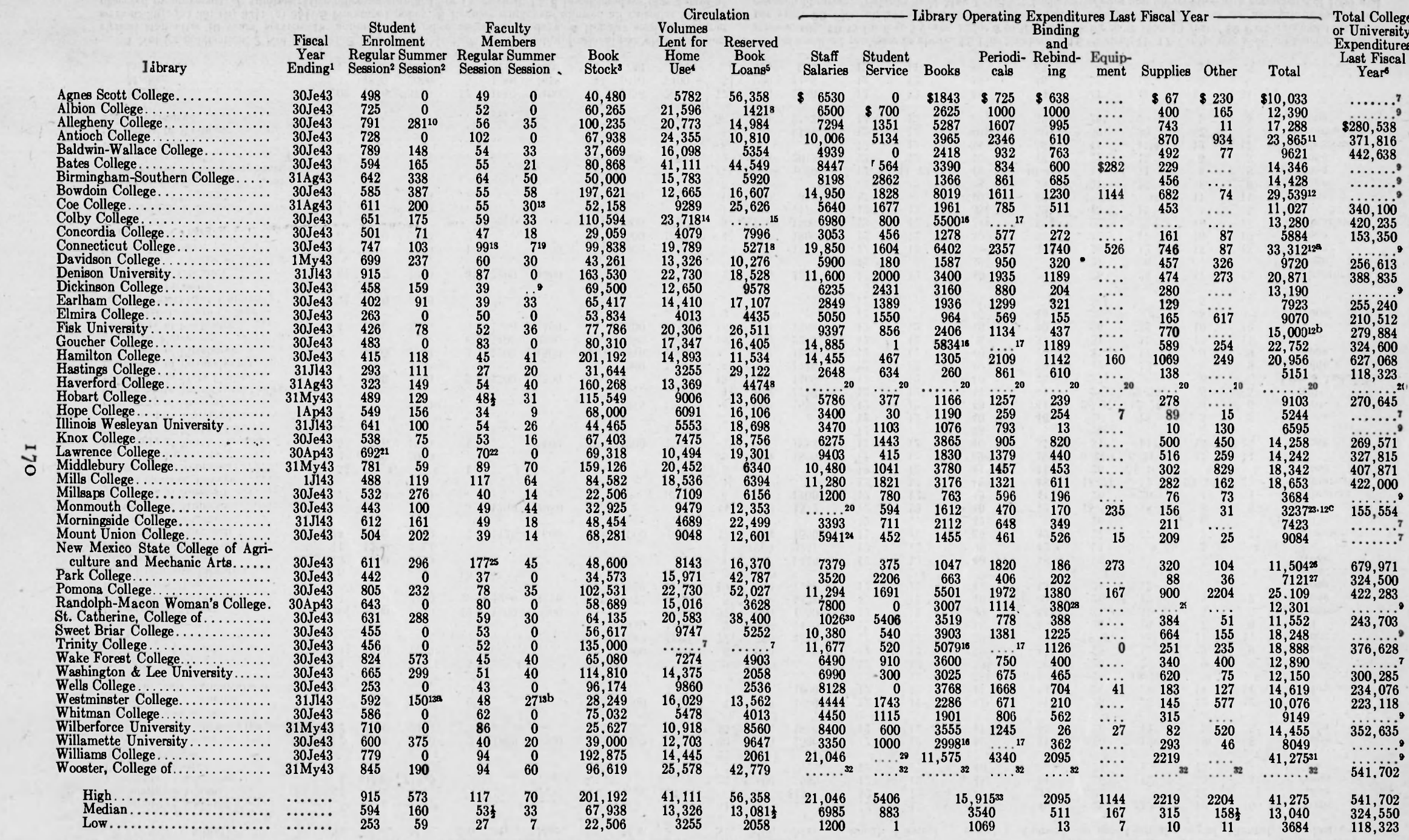

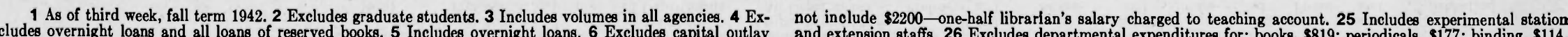

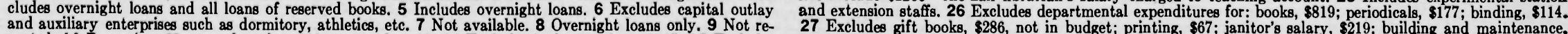
and auxiliary enterprises such as dormitory, athletics, etc. 7 Not available. 8 Overnight loans only. 9 Not re-
ported. 10 Presession, 93; second session, 229; postsession, 10. 11 Excludes janitor's salary, \$315. 12 Excludes ported. 10 Presession, 93; second session, 229; postsession, 10. 11 Excludes janitor's salary, \$315. 12 Excludes
building operation and maintenance expense: $\$ 2568 ;$ (a) $\$ 2832$; (b) $\$ 66 ;$ (c) $\$ 2040.13$ Summer session; second

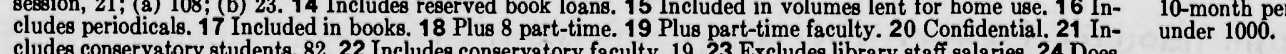

27 Excludes gift books, \$286, not in budget; printing, \$67; janitor's galary, $\$ 219 ;$ building and maintenance, services of Sisters of St. Joseph evaluated at \$3046. 31 Excludes expenditures iur: Chapin Library, \$5916. 32 For 
College and University Library General and Salary* Statistics (Group II)**

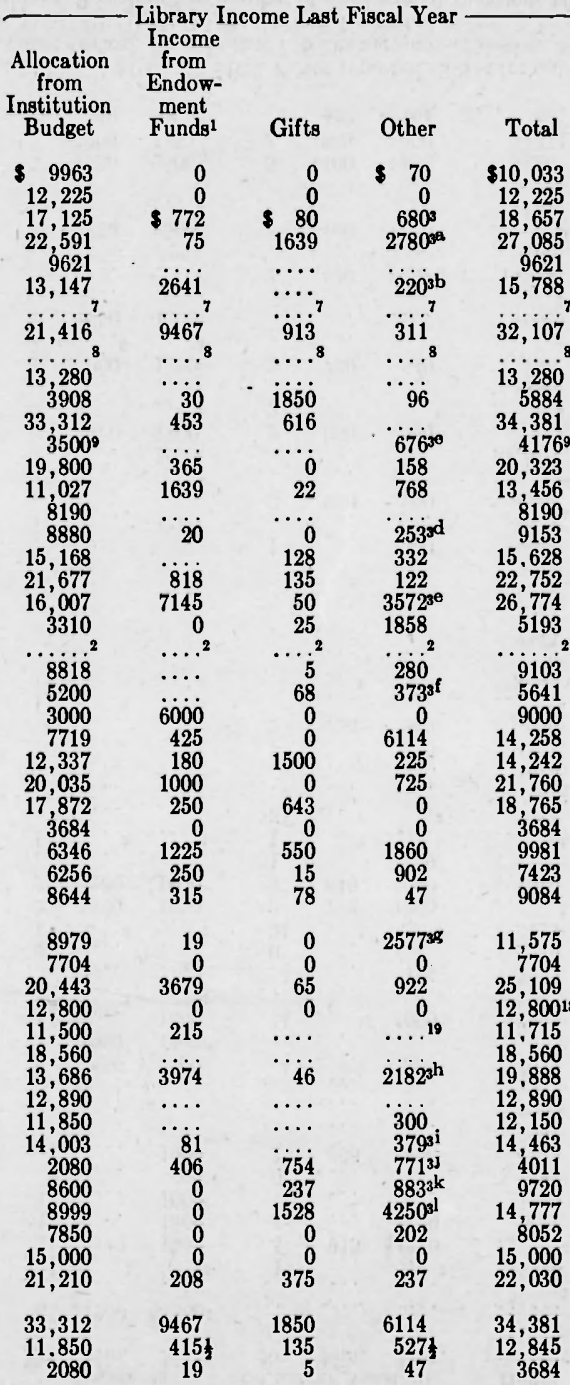

\author{
Number of Employees
in Full-Time Equivalent
Subpro- Admin-
fessional, istrative
Pro- Clerical, Office
}

Library

Institution ment

Agnes Scott College.

Allegheny Colle

Antioch College...............

Bates College.

.

Colby College.

Concordia College.

Connecticut College.

Denison University.

Dickinson College.

Elmira College.

Fisk University.

Hamilton College

Haverford College.

Hobart College .

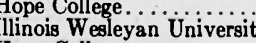

Knox College.

Lawrence College......

Mills College....

Monmouth Colleg.

Morningside College.

Tew Mexico State Coli...............

culture and Mechanic Ar

Park College

Pomona College.......

Randolph-Macon Woman's Colle

Sweet Brine, College of .

Trinity College.

Washington \& Lee University

Wells College ............

Whitman College...

Wilberforce University

illiams College.

High......
Median
Low

1 Includes trusts and all invested funds specifically designated for library purposes. 2 Confidential. 3 In (i) \$324; (j) \$123; (k) \$541; (l) \$96. 4 Not for publication. 5 Plus for summer session: $\$ 100$; (a) $\$ .75$ per hr. (b) $\$ 300 ;$ (c) $\$ 250$; (d) $\$ 130$; (e) $\$ 200$; (f) $\$ 103.6$ Acting librarian. 7 Not reported. 8 No separate college budget. 9 Does not include allocation for salaries. 10 Acting librarian also head cataloger. 11 Paid by Meharry Medical
College. 12 Part-time acting librarian not included. 13 Not full-time librarian. 14 Plus $\$ 50$ for 1 course or $\$ 200$

\begin{tabular}{|c|c|c|c|c|c|c|}
\hline $\begin{array}{c}\text { Chief } \\
\text { Librarian }\end{array}$ & $\begin{array}{c}\text { Associate } \\
\text { or } \\
\text { Assistant } \\
\text { Chief } \\
\text { Librarian }\end{array}$ & \multicolumn{2}{|c|}{$\begin{array}{l}\text { Administrative } \\
\text { Office Assistants }\end{array}$} & \multicolumn{2}{|c|}{$\begin{array}{l}\text { Department } \\
\text { No. Min. }\end{array}$} & $\begin{array}{l}\text { Heads } \\
\text { Max. }\end{array}$ \\
\hline $52400^{2}$ & $\cdots$ & *. & ... & 2 & & \\
\hline $200^{4}$ & $s 760$ & .. & .... & " & $\$ 1700$ & $\$ 1800$ \\
\hline $\begin{array}{l}2560 \\
2700^{5}\end{array}$ & $\$ 1760$ & 1 & $\$ 1275$ & . & $\cdots$ & ... \\
\hline $2800^{\circ}$ & 1800 & .. & $\ldots$ & 2 & 1000 & $\begin{array}{l}1400 \\
1500\end{array}$ \\
\hline 5278 & 3878 & i & 1260 & & $10 \angle 0$ & $\cdots$ \\
\hline 2900 & 1800 & 1 & 780 & 1 & … & 1500 \\
\hline $\begin{array}{l}2400 \\
3900\end{array}$ & $\begin{array}{l}1140 \\
\ldots \ldots\end{array}$ & . & $\ldots$ & $\ddot{4}$ & 1900 & 2400 \\
\hline $\begin{array}{l}3000 \\
23006\end{array}$ & $1500^{\mathrm{s}}$ & $\ddot{0}$ & $\cdots$ & $\ddot{2}$ & 1500 & $1600^{6}$ \\
\hline${ }^{2}$ & .... & .. & …. & & $\cdots$ & \\
\hline 1650 & $\cdots$ & $\because$ & $\cdots{ }_{4}$ & 1 & $\cdots$ & 1000 \\
\hline$\ldots 2,10$ & $\ldots{ }^{2,11}$ & .. & …" & $\begin{array}{l}0 \\
3\end{array}$ & $\cdots{ }_{2}$ & $\ldots{ }^{\prime}$ \\
\hline $3300_{6.2}$ & 1560 & $\mathrm{i}$ & 1080 & $\begin{array}{l}3 \\
1\end{array}$ & 2000 & $\begin{array}{l}2600 \\
1600\end{array}$ \\
\hline$\ldots{ }^{6.7}$ & & & & 1 & … & \\
\hline$\ldots{ }^{7}$ & $\ldots{ }^{7}$ & i & $\ldots^{7}$ & 3 & $\ldots^{7}$ & $\ldots{ }^{7}$ \\
\hline $2000^{6.7} 5^{6}$ & $1400^{7}$ & $\because$ & $\cdots$ & $\because$. & $\ldots$ & $\ldots$ \\
\hline 2000 & & $\because .$. & .... & ii & & 1320 \\
\hline 32006 & $\ldots$ & .. & $\ldots$. & 2 & 1500 & 1500 \\
\hline$\cdots_{B .2}^{2}$ & $\cdots$ & $\because \mathrm{i}$ & $\cdots_{2}$ & $\begin{array}{l}3 \\
3\end{array}$ & 1250 & 1850 \\
\hline & & 1 & $\ldots{ }^{2}$ & .. & ..... & ..... \\
\hline 13 & $1200^{14}$ & .. & $\ldots$ & .. & $\ldots$ & $\ldots$ \\
\hline$\ldots^{2}$ & $1200^{15}$ & .. & $\cdots$ & .. & $\cdots$ & $\cdots$ \\
\hline 4400 & 1800 & ... & .... & i & ..... & $1300^{10}$ \\
\hline 2640 & $\ldots$. & .. & $\ldots$ & 2 & 1620 & 2160 \\
\hline $\begin{array}{l}2190 \\
3250\end{array}$ & 2500 & i & $\dddot{900}$ & i & $\ldots$ & 1900 \\
\hline$\ldots 2^{2}$ & $\ldots$ & .. & $\ldots$ & i & .... & \\
\hline 3000 & 2000 & $\mathrm{i}$ & 1200 & & $\cdots$ & \\
\hline $1800^{3} \mathrm{c}$ & $540^{5 \mathrm{~d}}$ & i & $\ldots .{ }^{7}$ & $\begin{array}{l}2 \\
1\end{array}$ & $\ldots^{7}$ & $1400^{5}$ \\
\hline $2250^{\circ}$ & $\ldots$ & .. & $\ldots$ & $\mathrm{ij}$ & $\ldots$ & …, \\
\hline 1800 & … & .. & … & $\begin{array}{l}1 \\
. .\end{array}$ & $\ldots$ & $\ldots{ }^{2}$ \\
\hline $1900 \mathrm{f}$ & $\ldots 1^{15^{\mathrm{A}}}$ & 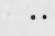 & $\cdots$ & $\ddot{3}$ & 1320 & 1760 \\
\hline 2000 & 1350 & $\because$ & 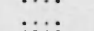 & & & \\
\hline $\begin{array}{l}4000^{6} \\
\ldots 2^{2}\end{array}$ & $\ldots$ & 1 & 1300 & $\begin{array}{l}2 \\
4\end{array}$ & $\begin{array}{l}2500 \\
\ldots{ }^{2}\end{array}$ & 3200 \\
\hline 5278 & 3878 & 1 & 1300 & 4 & 250 & 3200 \\
\hline $\begin{array}{l}2640 \\
1650\end{array}$ & $\begin{array}{r}1560 \\
540\end{array}$ & 1 & 1200 & 2 & 1500 & 1600 \\
\hline & & & & & & \\
\hline
\end{tabular}

for 4 courses. 152 assistant librarians: confidential; (a) minimum \$1440. maximum \$1620. 1610 months only. 17 Pomona, Scripps, and Claremont Colleges operate joint order and catalog department employing 5 employees for joint organization - not included. 18 Salaries, books, periodicals, and binding only; other items on general college budget. 19 Excludes evaluation of Sisters' services, \$3046. 20 Sisters; no salaries; services evaluated at colleges and universities with enrolments under 1000 . 


\section{College and University Library General and Salary* Statistics (Group II)**}

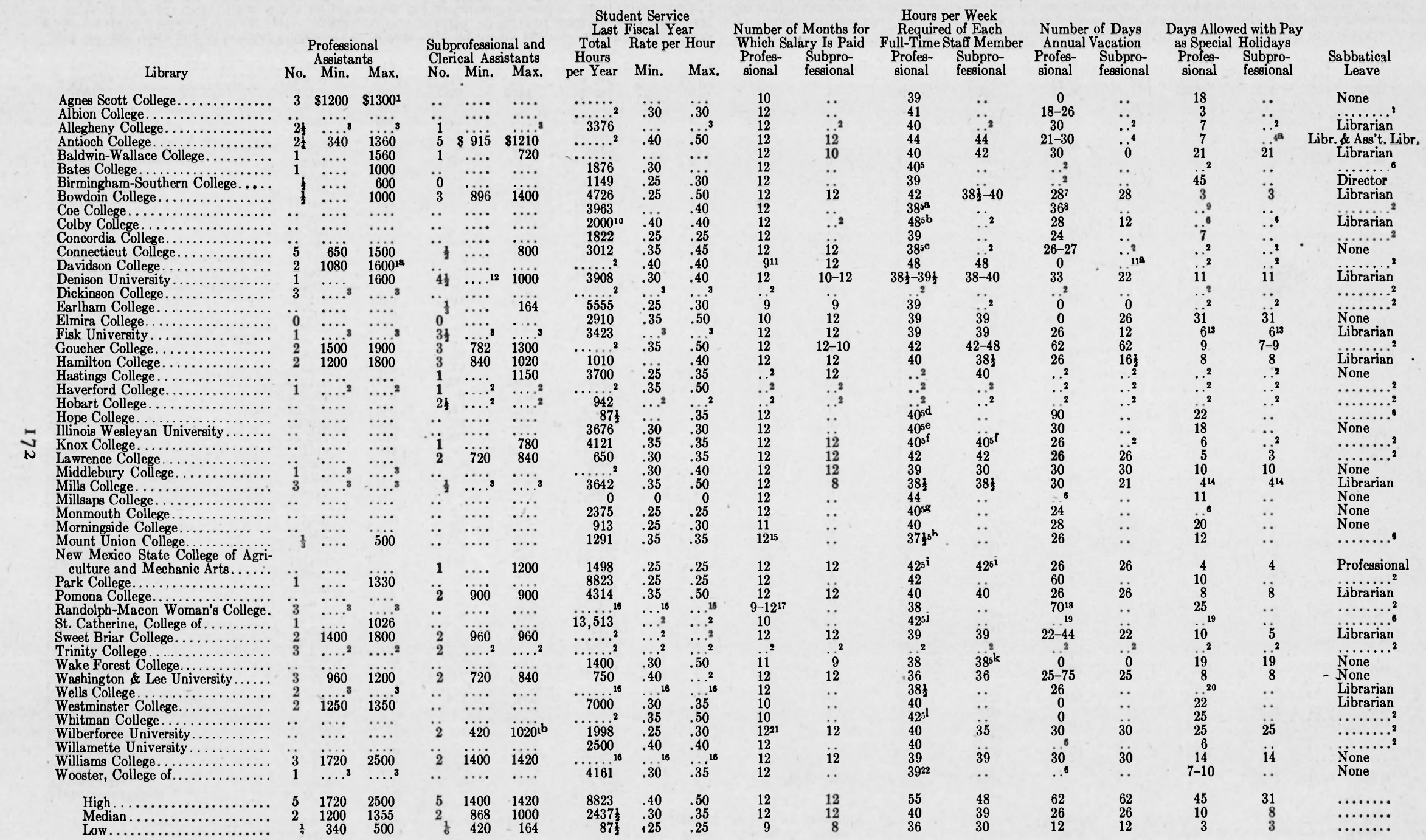

1 Plus salary for summer session: $\$ 130$; (a) $\$ 100$; (b) $\$ 103.2$ Not reported. 3 Not for publication, or confidential. 4 Subprofessional and others, none; clerical, 21; (a) none; 7. 5 During summer session, 36; (a) 33; (b) 301 8 Includes number of days special holidays. 9 Included in number of days annual vacation. 10 Summer session

per month for 10 months: $\frac{1}{2}$ clerical on hourly rate. 13 Plus part of each recess. determined vearly. 14 Plus 1 week at Christmas. 15 Except department head, 10 months only. 16 Not included in library budget. 17 Librarian, 12;
others, 9.18 Librarian only 19 Does not apply to Sisters; 1 secular on 10 months basis. 20 About one month. 213 for 12 months, 1 for 10 months. 2239 hours for 5 staff members; 30 hours for $1 ; 31$ hours during summer
session. ${ }^{*}$ Salaries as of May 1,1943 . ${ }^{*}$ Includes colleges and universities with enrolments under 1000 


\section{Teachers College and Normal School Library General and Salary Statistics}

Library

Arizona, Flagstaff, State Teachers College. Arkansas, Conway, State Teachers College California, San Diego, State College ................
Colorado, Greeley, State College of Education State Teachers Collese.

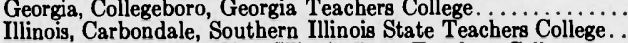
Illinois, DeKalb, Northern Illinois State Teachers College Kansas, Emporia, State Teachers College.

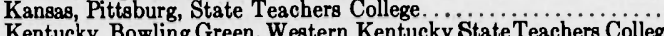
Kentucky, Bownig Green, Westen Kentucky state Teachers Collese Minnesota, Bemidji, State Teachers College. Minnesota St. Cloud State Teachers College.

Minnesota, Winona, State Teachers College. Mississippi, Cleveland, Delta State Teachers Colli........ Missour, Cape Girardeau, Southeast Missouri State Teachers Colleg. .

. ebraska, Kearney, State Teachers College

New Mexico Las Vegas, Highlands University...

New York, Albany, State College for Teachers. New York, Geneseo, State Normal School.

North Dako, Valley Cit, State Teachere College Colle.....

Orthom Ed

Oregon, Ashland, Southern Oregon College of Education...

Oregon, La Grande, Eastern Oregon College of Education....

Oregon, Monmouth, College of Education......................

Pouth Dakota, Aberdeen, Northern State Teachers College.

Texas, Denton, North Texas State Teachers College ...........

Texas, San Marcos, Southwest Texas State Teachers College........

Virginia, Farmville, State Teachers College

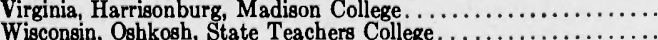

Wisconsin, Stevens Point, Central State Teachers College...........

High ...........................................

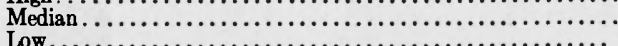

$1 \mathrm{As}$ of third week, fall term 1942; includes graduate students. 2 Includes graduate students. 3 Includes volumes in all agencies. 4 Excludes overnight loans and all loans of reserved books. 5 Includes overnight loans
only. 6 Excludes capital outlay and auxiliary enterprises such as dormitory, athletics, etc. 7 First session. Second ons. 6 : 23 ; (a) 173 ; third term, 75 ; (b) second term, 9 ; third term, 6 ; (c) 194 ; (d) 32 ; (e) 235 ; (f) 11 ; (g) 243 ;

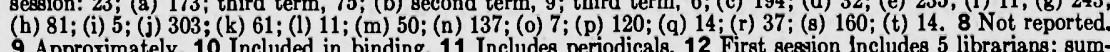
mer session includes 3 librarians; (a) 4 librarians; (b) 1 librarian. 13 Excludes building operation and maintenance:

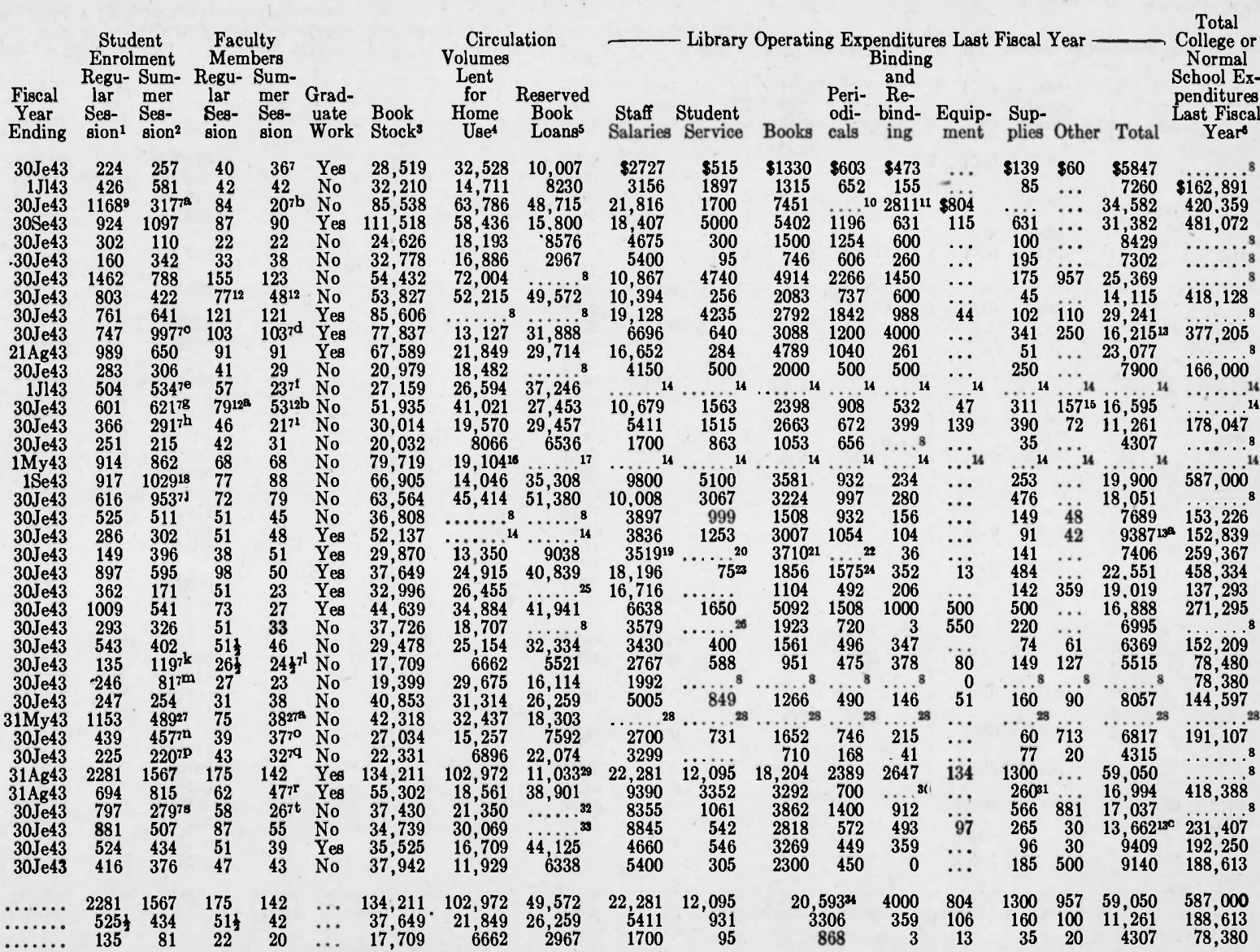

$\$ 3916$; (a) $\$ 1585$; (c) $\$ 962.14$ Not available. $15 \$ 62$ for printed catalog cards. 16 Total circulation; Includes reserve book loans. 17 Included in volumes lent for home use. 18 Special August session, 143.19 Includes student service. 20 Included in staff salaries. 21 Includes periodicals. 22 Included in books. 23 Summer seasion only.
24 Tro years' periodical expenditure. 25 Open shelves, no record kept. 26 Not paid from library funds. 27 Presession, 286; postsession, 210; (a) presession, 25; postsession, 21.28 Confidential. 29 Includes overnight loans only. 30 Included in salaries and supplies. 31
$50,000.34$ Totals for books and periodicals. 


\section{Teachers College and Normal School Library General and Salary* Statistics}

Library

Arizona, Flagstaff, State Teachers College. Arkansas, Conway, State Teachers College. Colorado, Greeley, State College of Education.......... Connecticut, New Haven, State Teachers College... , Carbondale, Southern Illinois State Teachers College.. , Northern Illinois State Teachers College ........... Kansas, Emporia, State Teachers College. . . Kansa, Pittoburg, State Teachers College........... Kinch Minnesota, Bemidji, State Teachers College . Minneso Mississippi, Cleveland, Delta State Teachers College... Missouri, Cape Girardeau, Southeast Missouri State Teachers College Cape Girardeau, Southeast Missouri State Teachers College Missouri, Warrensburg, Central Missouri State Teachers College. . . Nebraska, Kearney, State Teachers College......

New Mexico, Las Vegas, Highlands University ...

New York, Alban, State College for Teach

New York, Geneseo, State Normal School.

Carth Carolina, Greenville, East .Carolina Teachers College... Okrth Dakota, Valley City, State Teachers College.

Oregon, Ashland, Southern Oregon College of Education ....

Oregon, La Grande, Eastern Oregon College of Education.

Oregon, Monmouth, College of Education.

Pennsy vania, West Chester, State Teachers College

South Dakota, Aberdeen, Northern State Teachers College....

Douth Dakota, Spearfish, Black Hills Teachers College..

Texas, San Marcos, South west Texas State Teachers Colleg.

Virginia, Farmville, State Teachers College

Wisconsin

Wisconsin, Stevens Point, Central State Teachers College..........

Migh. .

Library Income Last Fiscal Year
Allocation Allocation
from

Full-Time Equivalent

Profor Subpro- Clerical rofes- Subpo- and Chief Assistant

Subprofessional and

Budget Other Total

\begin{tabular}{|c|c|c|}
\hline $\begin{array}{r}\$ 5704 \\
7260\end{array}$ & $\$ 43$ & $\$ 5747$ \\
\hline $\begin{array}{l}34,582 \\
\end{array}$ & & $\begin{array}{r}7260 \\
34,582\end{array}$ \\
\hline 29,554 & 5167 & 34,721 \\
\hline $\begin{array}{r}8429 \\
9100 \\
25,369\end{array}$ & 93 & $\begin{array}{r}8429 \\
9193 \\
25\end{array}$ \\
\hline 25,3 & $\ldots_{3}$ & $\begin{array}{l}25,369 \\
\ldots^{3}\end{array}$ \\
\hline$\ldots \ldots{ }^{4}$ & 293 & \\
\hline 17,632 & 2500 & 20,132 \\
\hline $\begin{array}{r}22,576 \\
7900\end{array}$ & 500 & $\begin{array}{r}23,076 \\
7900\end{array}$ \\
\hline$\ldots . .60$ & $\ldots_{6}$ & \\
\hline & $\ldots$ & \\
\hline $\begin{array}{r}11,261 \\
5000\end{array}$ & ${ }_{30}$ & $\begin{array}{r}11,261 \\
5030\end{array}$ \\
\hline 5000 & & \\
\hline 18,051 & & 18,051 \\
\hline 2400 & $3421^{7}$ & \\
\hline $\begin{array}{l}8801 \\
9400\end{array}$ & $3428^{7^{\mathrm{a}}}$ & 12,229 \\
\hline 21,971 & $614^{7 b}$ & $\begin{array}{r}9400 \\
22,585\end{array}$ \\
\hline 19,601 & $\cdots$ & 19,601 \\
\hline 16,788 & $\ldots$ & 16,788 \\
\hline $\begin{array}{l}0449 \\
5129\end{array}$ & $1241^{7 \mathrm{c}}$ & $\begin{array}{l}6369 \\
6369\end{array}$ \\
\hline 5648 & $62^{7^{\mathrm{d}}}$ & 57 \\
\hline 971 & $129^{7 e}$ & 8100 \\
\hline 8127 & $5341^{7 f}$ & 13,468 \\
\hline & $114^{78}$ & \\
\hline $\begin{array}{l}59,050 \\
16,994\end{array}$ & $\cdots$ & $\begin{array}{l}59,050 \\
16,994\end{array}$ \\
\hline 17,036 & …. & $\begin{array}{l}10,9 \\
17,0\end{array}$ \\
\hline & & 14614 \\
\hline 10,860 & 99 & 10,959 \\
\hline & & \\
\hline & 5341 & \\
\hline & 30 & \\
\hline
\end{tabular}

$6 \quad \$ 2580 \quad \$ 2940$

$\ddot{2} \quad \cdots r^{3} \quad \cdots{ }^{3}$

$\because 6 \quad 7750 \quad 1950$

$\ddot{6}_{6} \quad \cdots_{5} \quad \cdots_{5}$

$\cdots \cdots, \quad \cdots$,

$2 \quad \ldots c^{3} \quad \ldots{ }^{3}$

$\because \cdots, \quad \cdots \cdots$

i $\quad \cdots, \quad i 500$

. $\quad \cdots \quad \cdots$

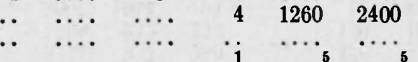

$\begin{array}{llllll}1 \frac{4}{5} & 2150 & 2741 & \because & 1650 & 1650 \\ 1 & \cdots & 23652 \mathrm{j} & 1 & \ldots & 3010\end{array}$

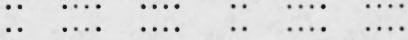

$\because \quad \cdots$

$\cdots \quad \cdots$

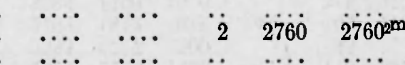

$2 \quad$ is50 2000

$2 \quad 1800 \quad 2100$

$\begin{array}{ll}1825^{2} & 1920^{2} \\ \cdots & \cdots\end{array}$

$\ddot{8} \quad 1500 \quad 2150$

$31600 \quad 1800$

$\begin{array}{lll}1 & \cdots & 1500 \\ 1 & \cdots & 1632\end{array}$

$\begin{array}{lll}2 & 1200 & 1632 \\ 2700\end{array}$

$\begin{array}{rllllr}6 & 2580 & 2940 & 8 & 2760 & 3010 \\ 2 & 1837 \frac{1}{2} & 2050 & 2 & 1575 & 1800 \\ 1 & 1750 & 1500 & & 1200 & 900\end{array}$

of $\$ 2341$; (a) $\$ 1468$; (b) $\$ 19$; (c) $\$ 56$; (d) $\$ 62$; (e) $\$ 129$; (f) $\$ 1666$; (g) $\$ 13.82$ associate or ascistant chief librarians: \$3171; \$3279. * Salaries as of May 1, 1943 . $\begin{array}{rrrrr}51500 & \$ 2000 & 3 & 720 & 1140\end{array}$ $\begin{array}{lllll}1200 & 1500 & \ldots & \ldots & \ldots\end{array}$

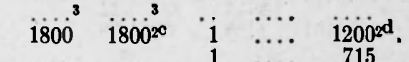
$\ldots{ }^{5}$ ... $\cdots$ 600 691 $550^{2 k}$
.. $\quad \cdots \quad \cdots$

Clerical Assistants $\cdots \cdots$
1 Acting librarian. 2 Plus $\$ 275$ for summer session; (a) $\$ 192$; (b) $\$ 478$; (c) $\$ 400$; (d) $\$ 267$; (e) $\$ 329$; (f) $\$ 314$;
(g) $\$ 286$; (b) $\$ 265$; (i) $\$ 325 ;$ (i) $\$ 300 ;$ (k) $\$ 200$; (1) $\$ 16$; (m) $\$ 150$; (n) $\$ 500.3$ Not reported. 4 No separate budget for the library. 5 Not for publication or 


\section{Teachers College and Normal School Library General and Salary Statistics}

\section{Library}

Arizona, Flagstaff, State Teachers College

Arkansas, Conway, State Teachers Collese

Colorado, Greeley, State College of Ėducation

Connecticut, New Haven, State Teachers College.

Georgia, Collegeboro, Georgia Teachers College.

.

Kansas, Emporia, State Teachers College

Kentucky, Bowling Green, Western Kentucky State Teachers Colleg

Minnesota, Bemidji, State Teachers College.

Minnesota, Mankato, State Teachers College

Minnesota, St. Cloud, State Teachers College.

Msisoippi, Cle College

Missouri, Warrengburg Central Missouri State Teachers College.

Nebraska, Kearney, State Teachers College . . .

Nebraska, Peru, State Teachers College..........

New Mexico, Las Vegas, Highlands University

New York, Albany, State College for Teach

North Carolina, Greenville, East Carolina Teachers College.

North Dakota, Valley City, State Teachers College.

Oklahoma, Edmond, Central State Teachers College.......

Oregon, Ash Gran, Southern Oregon College of Education.

Oregon, Monmouth, College of Education

Pennsylvania, West Chester, State Teachers College..........

South Dakota, Spearfin, Blak Hills Teachers Cors Colle

Texas, Denton, North Texas State Teachers College.

Texas, San Marcos, Southwest Texas State Teachers College

Virginia, Farmville, State Teachers College...

Virginia, Harrisonburg, Madison College.

Wisconsin, Stevens Point Central State Te ..................

High. ...

Lew.......

Student Service Last

Fiscal Year
Total
Rate per Hour

$\begin{array}{ccc}\text { Hours } & \text { Mini- } & \text { Maxi- } \\ \text { Yorar } & \text { mum } & \text { mum }\end{array}$

$1058 \quad \ldots 1 \quad \ldots$

$\begin{array}{ccc}\ldots 358 & .60 & .60 \\ 14,983 & .30 & .40\end{array}$

$\begin{array}{lll}\ldots 1 & .25^{1} & .25^{1}\end{array}$

13,107

13,107
$\cdots \cdots \cdots 1$
$\cdots, m^{1}$

213

1134
2300

2300
1160
6091

500

500
7612

7612
$\ldots{ }^{1}$

12,268
2854
4661

4661
0
$250^{4}$

$250^{4}$
0
$5940^{5}$

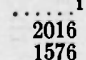

358
1989

184

4813
40,318
11,172

40,318
11,172
3500

…1 1365

$1016 \quad .40$

40,318
285
250
Number of Months for

Which Salary Is Paid
Subpro-

Profes. fessional

sional Clerical

列
Hours per Week Required of Each
Full-Time Staff Member

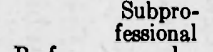

Profes-
sional Clerical

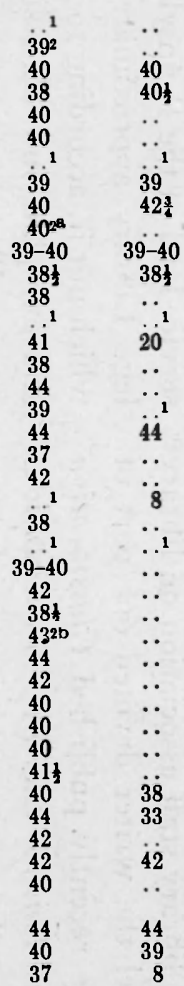

Number of Days Allowed with Pay as:

$\begin{array}{ccc}\begin{array}{c}\text { Subpro- } \\ \text { fessional } \\ \text { and }\end{array} \text { Profes- } & \begin{array}{c}\text { Subpro- } \\ \text { fessional }\end{array} \\ \text { Profes- } & \text { and }\end{array}$

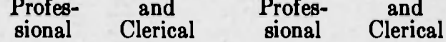

14
27
7
11
18
11
18
201
5
11
22
20
11
51
12
10
15
4
24
4
25
26
11
40
5
6
14
10
4
20
11
4
12
12
10
18
5
18
40
13
4
Sabbatical

$\ldots \ldots{ }^{1}$

None

None

None

Ail

Professional

All

All

Ail ${ }^{\prime . . .}$

Aill

Professional

None

None

Not for the duration

None

Librarian

Librarian

None

None

None

None

. none

None

….......

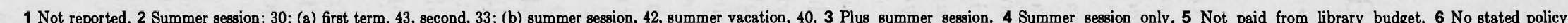
7 1 Not reported. 2 Summer session: 30 ; (a) frst term, 